\title{
Sutureless and Glue-Free Conjunctival Autograft in Pterygium Surgery: A Case Series
}

\author{
Md. Ameenur Rashid Akanda, ${ }^{1}$ Yousuf $A l i,{ }^{2}$ Sharmin Ferdousi ${ }^{3}$
}

\begin{abstract}
Foreign materials used in ocular surface surgery may lead to local complications such as discomfort, scarring, or infection. Plasma-derived products such as fibrin glue may produce possible hypersensitivity reactions whereas the risk of viral transmission remains. We describe a simple method of achieving conjunctival autograft adherence during pterygium surgery avoiding potential complications associated with the use of fibrin glue or sutures.
\end{abstract}

Pterygium excision with limbal conjunctival autografting without using glue or sutures was performed in all the patients followed by bandaging for 24 hours. The patients were followed up post operatively on 2nd day, 1 week, 1 month, 3 month and 6 months. They were examined for haemorrhage, wound gape, graft shrinkage, chemosis, graft dehiscence, recurrence or any other complications.

Total 100 cases were included in this case series. Patient age in ranged from 23 to $\mathbf{7 8}$ years (mean 50. $08 \pm 12.76$ years). Surgical Time was (in Minutes) from 20-28; mean \& SD $23.76 \pm 1.89$. Total graft dehiscence occurred in 1 eye (1\%), graft retraction in 12 eyes (12\%) and recurrence was seen in 1 eye (1\%). At 3 month postoperatively, the gain in uncorrected visual acuity ranged from 2-3 line in Snellen chart in 13 eyes. No other complication was noted.

This simple technique for pterygium surgery may prevent potential adverse reactions encountered with the use of foreign materials and in this case series provided safe and comparable results to current methods.

Key Words: Pterygium surgery, sutureless glue free, conjunctival autograft

TAJ 2019; 32: No-2: 56-61

\section{Introduction}

Pterygium is an abnormal overgrowth of fibrovascular tissue arising from the subconjunctiva toward the cornea. Although it can be easily excised, it has a high rate of recurrence ranging from $24 \%$ to $89 \% .{ }^{1}$ In 1985 , Kenyon et $\mathrm{al}^{2}$ proposed that a conjunctival autograft of the bare sclera could be used in treatment of recurrent and advanced pterygium. Recently, with the popularity of conjunctival autograft and use of antimetabolites such as mitomycin $\mathrm{C}$ and 5Fluorouracil the incidence of recurrence has been greatly reduced up to $12 \%{ }^{3-5}$ The role of carbon dioxide and eximer lasers in pterygium surgery remains uncertain.

Therefore, a simple surgical procedure that can reduce the recurrence rate to an acceptable level with minimal complications and without the use of potentially toxic drugs or radiotherapy would be ideal for the management of pterygium.

\footnotetext{
${ }^{1}$ Senior Consultant, General Hospital, Pabna.

${ }^{2}$ Associate Professor, Department of Ophthalmology, Rajshahi Medical College, Rajshahi.

${ }^{3}$ Assistant Professor, Department of Pathology, Rajshahi Medical College, Rajshahi.
} 
Sutureless grafting has been used successfully in gingival grafts, ${ }^{6}$ and represents a similar mucosal membrane tissue environment to the conjunctiva of the eye.

\section{Aims \& Objectives}

Foreign materials used in ocular surface surgery may lead to local complications such as discomfort, scarring or infection. Plasma-derived products such as fibrin glue may produce possible hypersensitivity reactions whereas the risk of viral transmission remains. We describe a simple method of achieving conjunctival autograft adherence during pterygium surgery avoiding potential complications associated with the use of fibrin glue or sutures.

\section{Materials and Methods}

The study sample was comprised of 100 eyes of 100 patients with primary pterygium underwent surgical procedure in Pabna General Hospital, Pabna \& Makka Eye Hospital, Rajshahi. Study was conducted during the period of $1^{\text {st }}$ January 2017 to $31^{\text {st }}$ December 2018 for the period of 2 years. It was approved by the IRB, PMC \& Regional Director of Makka Eye Hospital. All clinical information was noted on structured proforma and consent was obtained from all patients.

Inclusion criteria: Patients of all ages and of either sex presenting with primary pterygium. Bilateral pterygium was not excluded.

Exclusion criteria: Recurrent pterygium, glaucoma, retinal pathology requiring surgical intervention, history of previous ocular surgery or trauma and were inability to complete the six month follow up period.

\section{Preoperative ophthalmic evaluation:}

Uncorrected and best corrected visual acuity, digital anterior segment photography, slit-lamp examination, fundoscopy and measurement of intraocular pressure were done in all patients.

\section{Surgical technique}

Peribulbar anaesthesia with 2\% lignocaine and $0.5 \%$ bupivacaine in $1: 1$ ratio was given preoperatively. Incision was made with spring scissor over pterygium in a line $1 \mathrm{~mm}$ medial to limbus cutting only cojunctiva then dissection of the body from the overlying conjunctiva in a smooth clear plane as possible using blunt and sharp dissection. The body of the pterygium was dissected $4 \mathrm{~mm}$ from the limbus down to the bare sclera. The subconjuctival pterygium tissue and the thickened segment of conjunctiva and adjacent Tenońs capsule were excised leaving bare sclera. Pterygium was removed from the cornea by avulsion. Then the size of bare scleral was measured with calipers and the area documented in $\mathrm{mm}^{2}$.

Graft was taken from the superior temporal quadrant of bulbar conjunctiva. The globe was rotated downwards with a forcep by assistant when necessary. For harvesting the conjunctival autograft, the four corners was marked with tip of the calipers the conjunctival limbal graft to be created $1 \mathrm{~mm}$ larger in width than the recipient bed but same as length. Two small opening was created at the limbal site and careful blunt dissection with Wescott tenotomy scissors was performed until the entire graft was free from Tenons reaching the limbus to include limbal stem cells that act as a barrier to the conjunctival cells migrating onto the corneal surface. Subsequently, the edges of the graft were cut by Vannas scissors. Forceps was used to gently slide the graft to the recipient bed with the epithelial side up and keeping the limbal edge toward the limbus.

Hemostasis was allowed to occur spontaneously with minimal use of cautery to provide autologous fibrin to glue the conjunctival autograft naturally in position without tension and the scleral bed was viewed through the transparent conjunctiva to ensure that residual bleeding did not lift the graft.

The graft was kept in position for $10 \mathrm{~min}$, In this 10 minute usually another pterygium surgery was started in another OT table to minimize operation time. After 10 minute the stabilization of the graft was tested with a Merocel spear centrally and on each free edge to ensure firm adherence to the sclera. The eye was bandaged for 24 hours.

Total surgical time was from first conjunctival cut to removal of lid speculum i.e. the time taken from 
the excision of pterygium to the time to secure the graft on the bed.

Post-operatively a pressure eye patch was applied. Analgesia was prescribed two times daily. Postoperative medication included Tab. ciprofloxacin $500 \mathrm{mg}$ bid for 5 days. T Mycin Plus Eye ointment (Aristovision, Bangladesh) three times daily was used for 1 week then gradual tapering for 3 weeks and liberal use of topical lubricating eye drops four times daily for 4 weeks. The patients were instructed to avoid rubbing their eyes and avoid dust, heat, direct sun exposure. The patients were also advised to wear sun glasses to reduce UVB exposure.

All patients were followed up after $24 \mathrm{~h}$, after 1 week, after one month, after three month, then after 6 month. Patients completed a questionnaire at each follow-up visit, especially during the first 3 visits grading pain, foreign body (F. B) sensation, photophobia, hyperemia and chemosis into four grades according to the intensity. The questionnaire was scored from (0 to 3)
0 = nothing; 1 = mild; 2 = moderate; 3 = severe . The data were collected as mean scores and recorded.

The main postoperative outcomes noted were the recurrence rate which was defined as fibrovascular proliferation invading the cornea more than $1.5 \mathrm{~mm}$ at the site of previously excised pterygium, graft dehiscence, graft retraction and the gain in uncorrected visual acuity (UCVA). The secondary outcomes were duration of surgery, postoperative pain, foreign body sensation, photophobia, hyperemia, chemosis, overall satisfaction and the complications as, persistent epithelial defect, dellen, inclusion cyst, pyogenic granuloma, conjunctival edema, corneo-scleral necrosis, infective scleritis, keratitis and endophthalmitis.

\section{Statistical analysis}

Data are expressed as mean \pm SD. Statistical analysis was performed using SPSS 16 for Windows (IBM Corp., New York, NY, USA).

\section{Results}

Case series was carried out in 100 consecutive eyes with primary pterygium.

\section{Table 1 Demographic Profile of the Study Population}

\section{Demographic Data}

Range of age in (years)

Mean age in (years)

Sex

Males

Females

Laterality

Right

Left

Site of pterygium

Only Nasal

Nasal +Temporal

Size of pterygium in mm length mean \& SD

Surgical Time in minutes mean \& SD $(\mathbf{n}=100)$

23-78

$50.08 \pm 12.76$

38

62

58

42

96

04

$4.398 \pm 1.534$

$23.76 \pm 1.89$ 
Table 2: Showing postoperative main and secondary outcomes.

\begin{tabular}{|c|c|}
\hline Recurrence rate & $1(1 \%)$ \\
\hline Graft dehiscence & $1(1 \%)$ \\
\hline Early graft retraction & $12(12 \%)$ \\
\hline Medial side & $10(10 \%)$ \\
\hline Upper side & $2(2 \%)$ \\
\hline Early Graft oedema & $14(14 \%)$ \\
\hline \multicolumn{2}{|l|}{ Haemorrhage underneath the graft } \\
\hline On $1^{\text {st }}$ Follow up & $20(20 \%)$ \\
\hline On 2nd Follow up & $4(4 \%)$ \\
\hline On 3rd Follow up & $0(0 \%)$ \\
\hline Gain in UCVA & $13(13 \%)$ \\
\hline Conjunctival edema & $6(6 \%)$ \\
\hline Conjunctival granuloma & $0(0 \%)$ \\
\hline Corneal scar (faint nebula) & $8(8 \%)$ \\
\hline Dellen & $0(0 \%)$ \\
\hline Operative time in minutes (mean, SD) & $23.76 \pm 1.89$ \\
\hline Conjunctival cyst & $0(0 \%)$ \\
\hline Graft necrosis & $0(0 \%)$ \\
\hline Symblepharon & $0(0 \%)$ \\
\hline Scleral necrosis & $0(0 \%)$ \\
\hline Scleral thinning & $0(0 \%)$ \\
\hline
\end{tabular}

The gain in uncorrected visual acuity (UCVA) occurred 3 months post operatively and ranged from 2-3 line in 13 eyes. All cases with a gain in UCVA were due to clearance of visual axis occupied by pterygium pre-operatively.

Conjunctival edema occurred in 6 eyes (6\%). Most cases of conjunctival edema resolved gradually within the first post-operative week. Faint corneal nebula occurred in eight eyes (8\%) 
Figure 1: Nasal pterygium before \& after surgery, after 1 month \& after six month

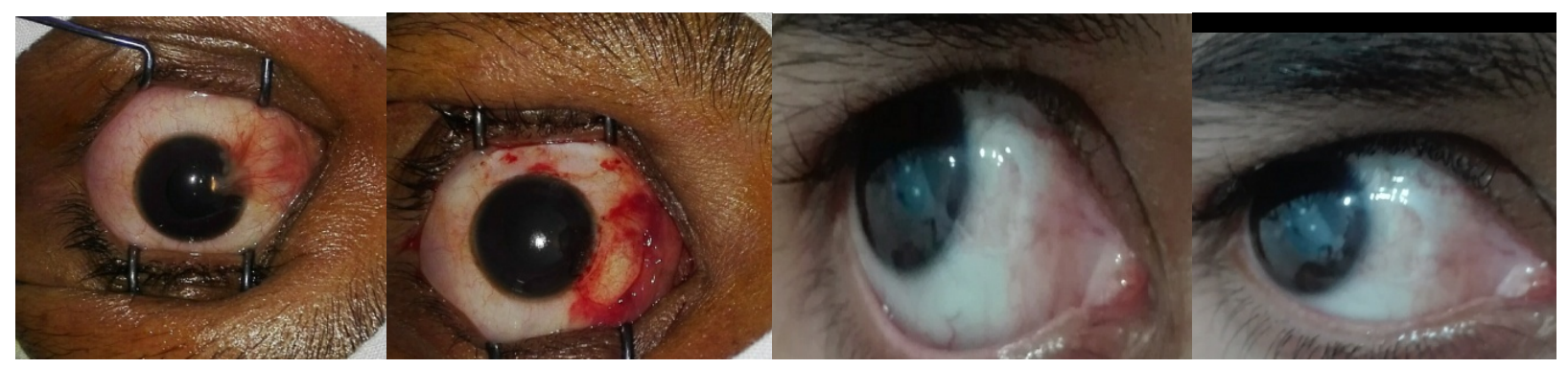

\section{Discussion}

While performing surgery the surgeon should keep in mind the possibility of more aggressive recurrent pterygium, so that the operative procedure chosen should be such that it minimizes recurrence. Hence, the aim of pterygium surgery should not only be excision of pterygium but also prevention of its recurrence. Generally, the pterygium recurrences occur within the first 6 months after surgery. ${ }^{7}$ One such method to prevent recurrence is autologous limbal conjunctival grafting. Limbal conjunctival autograft transplantation re-establishes the barrier function of limbus and hence significantly lowers the recurrence rate.

In this study the recurrence rate (1\%). Massaoutis et al. ${ }^{8}$ stated that the concept of surgical success in pterygium surgery can be defined as the provision of a white cosmetic conjunctiva, with no persistent symptoms and a low recurrence rate (less than $10 \%)$. The recurrence rate in our study agrees with The Massaoutis et al.'s criteria. The recurrence rate is also similar to Malik et al. ${ }^{9}$ who reported recurrence rate of $2.5 \%$ using a similar procedure of sutureless and glue free graft.

Graft dehiscence is a recognized complication of techniques using glue. ${ }^{10,11}$ Froutan et al. ${ }^{12}$ reported $13.33 \%$ rate of graft dehiscence using autologous fibrin and attributed this to a low concentration of thrombin and fibrinogen in autologous glue compared to a commercial preparation. In our study graft dehiscence occurred in 1 eyes (1\%) that was due to patient rubbing his eye vigorously. Hence, we instruct patients to use a protective glass and not to rub the eye in the 1st week post- operatively. Additionally, meticulous dissections of thin donor limbal conjunctival autograft free of Tenons capsule are mandatory for successful graft uptake.

Graft retraction was reported by $\operatorname{Tan}^{13}$ who advocated sub-conjuctival fibrosis and recommended meticulous dissection of subepithelial graft tissue. Foroutan et al. ${ }^{12}$ reported $20 \%$ of cases with graft retraction, in our study graft retraction occurred in 12 eyes out of 100 (12\%) eyes, among them 10 from medial side and 2 from upper side. All the cases of graft retraction were due to conjunctival chemosis and edema and were resolved with conservative treatment. In comparison, Wit et al. ${ }^{14}$ reported no graft displacement and postulated that sutureless and glue free graft resulted in even tension across the whole graft interface and no direct tension on the free edges resulting in reduced stimulus for subconjunctival scar formation. Wit et al. ${ }^{14}$ also proposed that the apposition of the eye lids to the bulbar conjunctiva provides a natural biological dressing, compression, and a smooth frictionless surface.

Pyogenic granuloma and dellen did not occur. Conjuctival edema occurred in our study in 6 eyes (6\%). All cases resolved spontaneously with conservative treatment.

The mean operative time in was $23.76 \pm 1.89 \mathrm{~min}$. This times are comparable however they are longer than other studies ${ }^{15,14}$ using fibrin glue which reported average operative time of $16 \mathrm{~min}$ (range 14-16) and 20 min (range 20-29) in suture group and reported $14( \pm 1.4)$ min in suture-less and glue free conjunctival autograft. But actually 
this time is much less if we reduct 10 minute while operating another case. None of our patients developed serious complications such as scleral necrosis, sclera thinning, graft necrosis, symblepharon, excessive bleeding, medial rectus muscle injury, or globe perforation.

\section{Conclusion}

Sutureless and glue-free limbal conjunctival autograft is safe, effective, economical, and its surgical outcomes following primary pterygium surgery are comparable to other procedure.

\section{References}

1. Gupta VP. Conjunctival transplantation for pterygium. DJO. 1997; 5:5-12.

2. Kenyon KR, Wagoner MD, Hettinger ME. Conjunctivalautograft transplantation for advanced and recurrent pterygium. Ophthalmology1985;92: 1461-1470.

3. Singh G, Wilson NR, Foster CS. Mitomycin eye drops as treatment for pterygium. Ophthalmology. 1988; 95:813-821.

4. Kleis W, Pico G. Thio-TEPA theory to prevent post operative pterygium occurrence and neovascularization. Am J Ophthalmol. 1973; 76: 371-373.

5. Tarr $\mathrm{KH}$, Constable IJ. Late complications of pterygium treatment. $\mathrm{Br} \mathrm{J}$ Ophthalmol. 1980; 64: 496-505.

6. Dorfman HS, Kennedy JE, Bird WC. Longitudinal evaluation of free autogenous gingival grafts. A four year report. J Periodontol. 1982; 53: 349-352.
7. Adamis AP, Starck T, Kenyon KR. The management of pterygium. Ophthalmol Clin North Am. 1990; 3: 611-23.

8. Massaoutis P, Khemka S, Ayliffe W. Clinical outcome study of a modified surgical technique for pterygium excision. Can J Ophthalmol. 2006; 41: 704-708.

9. Malik KPS., Goel R, Gupta SK, Kamal S, Malik VK, Singh S. Efficacy of sutureless and glue free limbal conjunctival autograft for primary pterygium surgery. Nepal J Ophthalmol. 2012; 4(8): 230-235.

10. Uy HS, Reyes JM, Flores JD, Lim-Bon-Siong R. Comparison of fibrin glue and sutures for attaching conjunctival autografts after pterygium excision. Ophthalmology. 2005; 112: 667-671.

11. Srinivasan S, Slomovic AR. Eye rubbing causing conjunctival graft dehiscence following pterygium surgery with fibrin glue. Eye. 2007; 21: 865-867.

12. Foroutan A, Beigzadeh F, Ghaempanah MJ, Eshghi $\mathrm{P}$, Amirizadeh N, Sianati H. Efficacy of autologous fibrin glue for primary pterygium surgery with conjunctival autograft. Iranian J Ophthalmol. 2011; 23: 39-47.

13. Tan D. Conjunctival grafting for ocular surface disease. Curr Opin Ophthalmol. 1999; 10:277-281.

14. Wit D, Athanasiadis I, Sharma A, Moore J. Sutureless and glue free conjunctival autograft in pterygium surgery: a case series. Eye. 2010; 24: 1474-1477.

15. Bahar I, Weinberger D, Dan G, Avisar R. Fibrin glue versus vicryl sutures for conjunctival closure. Cornea. 2006; 25(10): 1168-1172. 